Fecha de recepción: diciembre 2010

Fecha de aceptación: julio 2011

Versión final: marzo 2012

\section{Hacia las fronteras de la hiperrealidad social}

Fernando Luis Rolando Badell *

\begin{abstract}
Resumen: El presente trabajo se centrara en la influencia de lo virtual en el mundo real, considerando el estadio actual acerca de la alfabetización informática, la relación interfase-usuario e interfaz-espectador y como esta ha ido cambiando para facilitar el acceso masivo de las sociedades a los nuevos modos de comunicación y circulación de la información al otro lado de las pantallas, en una zona conocida como ciberespacio o hiperespacio considerando como esto podrá devenir en la construcción de nuevas tramas sociales, pero también afectar sus conductas. Para esto se partirá en tratar de analizar el problema de la interfase desde su génesis, tratando de explicar el mundo binario y las formas en que este puede construir lo que denominamos Hiperrealidad Social, como esto puede afectarnos socialmente, cuando el sujeto de estudio, en este caso el ser humano, se ve influido por la interacción que tiene como usuario mediante el uso de las interfase gráficas y la realidad virtual que opera al otro lado de las pantallas (detrás del cristal) en una zona que llamaremos ciberespacio, tratando de plantear en las conclusiones que aportes constructivos pueden hacerse al problema planteado.
\end{abstract}

Palabras clave: caminos - ciberespacio - concientización - evolución - hiperrealidad - interfase gráfica - matematización - mutación - objeto - observador - redes virtuales - relación - sensibilidad - simulacro - trama social - web 2.0 - web 3.0.

[Resúmenes en inglés y portugués en las páginas 110-111]

${ }^{(*)}$ Arquitecto (UB). Asistente en Diseño (UB). Especialización en Planeamiento (UB). Profesor de Artes Visuales (IUNA). Profesor Asociado de la Universidad de Palermo en el Departamento de Diseño de Imagen y Sonido de la Facultad de Diseño y Comunicación.

Una noche Zhuang Zhou soñó que era una mariposa: una mariposa que revoloteaba, que iba de un lugar a otro contenta consigo misma, ignorante por completo de ser Zhou. Despertóse a deshora y vio, asombrado, que era Zhou. Mas, ¿Zhou había soñado que era una mariposa? ¿O era una mariposa la que estaba ahora soñando que era Zhou? Entre Zhou y la mariposa había sin duda una diferencia. A esto llaman "mutación de las cosas..." (Zhuang, 1996) 1 


\section{Introducción}

\section{La alfabetización informática y la interacción con el mundo detrás del espejo}

Las tecnologías de la información han transformado nuestra forma de acceder al conocimiento. En su proceso de aprendizaje dentro del mundo digital, el usuario, espectador u observador que se sienta frente a una computadora realiza una serie de acciones, mediante un dispositivo del tipo ratón, pad, tableta gráfica, etc usando una interfase gráfica para realizar operaciones al "otro lado" del vidrio, pero aunque acceda a la información que busca, será su nivel de alfabetización informática, el que le diga si entiende o no lo que realmente esta haciendo.

Lo más interesante del caso es que si bien en muchas oportunidades el usuario puede realizar estas acciones en el mundo virtual moviéndose a través de él, casi siempre desconoce, que operaciones se están realizando en la computadora en segundo plano con la información que el usuario emite al realizar sus movimientos, sus huellas virtuales y cual si de un mundo al otro lado del espejo se tratase, puede ver a priori, lo que hace (su reflejo en ese espejo), pero no comprender en profundidad lo que ocurre dentro de esta interfase. En este sentido podemos decir que:

1. Toda tipo de interfase creada hasta hoy, es, en sí misma, solo un recorte de acceso al ciberespacio, es un perímetro, un universo limitado, que ha sido previamente diseñado por una compañía o diseñador y en el cual los grados de libertad que creemos tener dentro de la misma han sido decididos con anterioridad.

Por lo tanto, la noción de libertad esta siempre restringida a la evolución de los parámetros que constituyen esa interfase y los dispositivos que conectan al usuario con esta.

2. El observador modifica al objeto: cada usuario vive al acceder al ciberespacio (mundo virtual al otro lado de la pantalla) y operar dentro de él, una experiencia única e intransferible que depende de sus experiencias pasadas, sus conductas y del conocimiento pre-adquirido (alfabetización informática) que posea.

En este sentido, la alfabetización informática "real" en su mayor grado, que sería aquella en la cual los usuarios tuvieran los conocimientos suficientes brindados por los sistemas educativos desde que son niños, para no solo acceder, sino también para modificar, construir y comprender en profundidad el funcionamiento y las implicancias de actuar en el mundo virtual, esta recién en sus primeras etapas, dado que proyectos como el de Nicholas Negroponte o de muchas instituciones y gobiernos, están recién en sus comienzos y requerirán de décadas de desarrollo en donde el desafío a alcanzar será que las personas accedan al suficiente grado de alfabetización para ser capaces de generar libremente sus propias interfases, sus propias rutas, en lugar de solamente aprender a moverse por los caminos creados o los mundos creados por unos pocos que si tienen acceso a ese conocimiento, para dejar de ser meros consumidores y pasar a ser participes activos de su propio desarrollo.

3. El objeto modifica al observador: la información proveniente del ciberespacio puede modificar la forma de pensar y las conductas del observador, pudiéndolo afectar en grados diversos, 
ya sea desde dudar o creer en la información que se le transmite sobre un hecho vía internet, poniendo en crisis su noción de que es real y que no, hasta, haciéndolo ingresar a medida que los medios avanzan en un estadío llamado hiperrealidad, en donde en ciertos casos puede definir su estilo de vida, hábitos, relaciones, etc en función de como los medios redireccionan sobre él ciertos mensajes. En este sentido, la alfabetización informática debe incluir la discusión de estas problemáticas, para no transformarse únicamente en un tipo de alfabetización que solo enseña a manejar una herramienta, desconociendo el poder que esta tiene.

\section{La hiperrealidad y el mundo binario}

La Hiperrealidad es un termino que se utiliza dentro del mundo digital, para establecer un nivel de la conciencia en que resulta difícil diferenciar la realidad de la fantasía, especialmente cuando está potenciada a partir del uso de medios tecnológicos y de comunicación avanzados que "operan" sobre la mente del sujeto a partir de modelos de construcción binaria.

La construcción binaria posee en su propia génesis, como lenguaje que deviene del mundo matemático, la condición de operar con ceros y unos, con positivos o negativos, con luz y oscuridad, de pixeles prendidos o apagados, generando una trama de la realidad a partir de los procesos de digitalización de esta, que en el fondo es solo una ilusión.

Como lo binario, se trata en su origen de un lenguaje bipolar, que procede en su etapa fundacional de lo que se conoció en sus inicios como código máquina, dado que en su nivel más bajo es lo único que comprendían las computadoras, este proceso de matematización de la realidad posee la limitación de construir sus modelos de universo a esa escala.

Esto produce en su base, estructuras duales que generalmente son de suma utilidad en algunas áreas como por ejemplo el mundo financiero, dando lugar a sistemas que sirven para establecer ganancias o perdidas, altas o bajas, etc, pero que poseen algunas limitaciones al relacionarlos con el rico abanico de posibilidades que pueden tener las relaciones humanas dentro de las redes sociales.

De este modo, estas estructuras duales van estableciendo simplificaciones, o parametrizando los diferentes matices que pueden tener los seres humanos, en sistemas que aún son ciertamente rígidos y que en muchos casos pueden crear modelos maquinales a los cuales los usuarios deben adaptarse, y no al revés como sería más lógico. Un ejemplo en este sentido son las interfases que permiten al usuario a través de kioscos interactivos para hacer tramites financieros, que en muchos casos no son del todo ergonómicas y obligan a los bancos a que una persona real "instruya" al usuario acerca de su uso. Esto se debe a dos razones fundamentales:

En primer lugar a pesar de los avances alcanzados en los sistemas operativos en los últimas décadas en cuanto a velocidad y manejo de la información, el desarrollo de las interfases gráficas se encuentra aún utilizando prácticamente la misma estructura de metáfora de escritorio bidimensional que se conoce hace más de 25 años, a mediados de los 80 y recién en los últimos años ha comenzado el intento de popularización masiva de interfases tridimensionales que usen un sistema senso-touch, en donde el usuario prescinda del uso del ratón o mouse pad y acceda a los contenidos y se comunique con otros usuarios usando directamente sus propios dedos para mover y acceder a la información en las pantallas. 
En segundo lugar, a pesar de los avances en las redes de comunicación globales y del uso de internet y la world wide web, aún hoy existe una extensa cantidad de personas que superan cierta franja de edad, que no tienen posibilidades, o les resulta sumamente dificultosa la compresión o tienen cierta resistencia ideológica a la alfabetización informática, quedando de este modo fuera del acceso a las nuevas formas de comunicación que las redes brindan a la sociedad.

\section{Antecedentes}

\section{La relación interfaz espectador: el origen de las primeras interfases gráficas}

En el presente análisis se hace imprescindible para lograr una comprensión más detallada del problema de las interfases gráficas, conocer como surgieron estas.

A comienzos de la década del 60, en 1961, y de modo casi casual, un ingeniero informático de la Nasa, Douglas Engelbart, estaba aburrido durante una conferencia en donde se planteaba el tema de como manejar información gráfica con una computadora y se puso a realizar varios bocetos en su cuaderno de notas. En estos bocetos se le había ocurrido la idea de un dispositivo que fuera relativamente cómodo para el usuario y que permitiera mover los gráficos en la pantalla, inspirándose en un brazo mecánico que se usaba para medir superficies, con el que el ya había trabajado. Estos bocetos quedan allí, pasan tres años y en 1964, la NASA le pide a Engelbart que piense en qué métodos podían usar las personas que trabajaban en la central espacial para interactuar con sus computadoras, que para esa época eran máquinas que tenían el tamaño de una heladera y solo aceptaban tarjetas perforadas con ceros y unos (información binaria) como modo de cargar y acceder a la información. Así, retomó a esa idea y le pasa sus bocetos al ingeniero, Bill English, que construyó el primer prototipo en madera, que por su forma y porque tenia un cable (cola) que lo conectaba a la computadora terminó llamándose ratón (mouse). Este dispositivo permitía que una persona moviera desde algo externo a una pantalla (como el ratón), un cursor o puntero que estaba al otro lado de la pantalla y como herramienta de trabajo fue considerada de escasa utilidad en aquellos días dado que hasta ese momento los sistemas no habían desarrollado la interfase gráfica.

En el año 1973, un grupo de investigadores de Xerox en el Centro de Palo Alto en los Estados Unidos crea un propotipo no comercial que es usado para investigación en las universidades norteamericanas y que ya contenía en su génesis la idea de integrar el raton creado por Engelbart e English, pero sería recién con el lanzamiento del prototipo Xerox Star 8080, lanzado en 1981, en donde se desarrolló un primer sistema integrado llamado Xerox Star o Global View (Visión Global), que aludía a la metáfora en una pantalla de un escritorio con aplicaciones y una interfaz gráfica de usuario, que podía ser manejada desde afuera de esta con un ratón.

A pesar de lo revolucionario del sistema estos prototipos y los investigadores que lo desarrollaron tuvieron escaso apoyo de la compañía, hasta que dos jóvenes que pensaban, mientras estudiaban en la Universidad de Berkeley, que las computadoras debían estar al alcance de la gente común, como Steve Wosniak ( el hombre que desarrollo en su casa el primer prototipo de computadora personal y que se quemó) y Steve Jobs, un hombre con ideas revolucionarias sobre como humanizar los medios tecnológicos para hacerle la vida más sencilla a la gente, ambos fundadores de la empresa Apple, visitan la sede de Xerox en Palo Alto y convencen a los 
directivos de la empresa para que les muestren los prototipos.

Con estas ideas que habían nacido veinte años antes, mientras un ingeniero se aburría en una conferencia, Wosniak y Jobs desarrollan en Apple, el proyecto Lisa, que recibió este nombre en honor a la recién nacida hija de Steve Jobs, y que de algún modo, y no por casualidad a partir de este nombre, trato de humanizar la tecnología y brindó por primera vez la accesibilidad al manejo de las computadoras personales para cualquier persona, algo impensado pocos años antes. El primer sistema se llamo Apple Lisa Office System 1 (Lisa O.S), lanzado en 1983 y era un procesador de documentos de trabajo, que luego derivo en el Lisa SO 2 y el Lisa OS 7 en 1984, optimizando el sistema pero no la interfase gráfica de usuario, que recién evolucionaría con la llegada del proyecto Macintosh.

Por su parte, la empresa IBM, desarrolló en 1984 el VisiCorp. Esta fue la primera interfaz gráfica de escritorio para el PC de IBM, solo para las grandes corporaciones, dejando de lado el usuario común e incluía un ratón, tenía un instalador, un sistema de ayuda y no tenía iconos que sirvieran de metáfora de escritorio (por ejemplo un icono de una papelera, para que el usuario arrastre y tire allí lo que no usa), razón por la cual careció de masividad.

\section{El comienzo de la revolución de la interfaz gráfica}

Con la idea de poner la computadora personal al alcance de la gente común sin que esta tuviera grandes conocimientos de informática para poder usarla, en el año 1984, Steve Wosniak y Steve Jobs avanzan en la empresa Apple Computers en su proyecto Macintosh creando el sistema operativos Mac OS System 1.0, que tuvo la primera interfaz gráfica con varias características de un sistema operativo moderno, ya que utilizaba ventanas e iconos. Estas ventanas podían ser arrastradas con el ratón y los archivos y carpetas podían ser copiados o arrastrados por la pantalla a otro lugar, brindando herramientas al usuario para que pudiera manejarse de un modo sencillo, al otro lado de la pantalla, dentro del ciberespacio, siendo un estándar para el trabajo en diseño gráfico (desktop publishing) durante los años 80 .

\section{El primer nexo entre la interfaz gráfica y la televisión}

En 1985, Jay Glenn Miner de la empresa Didbit desarrollan la computadora Amiga 1000 creando el sistema operativo Workbench 1.0 , que poseía una interfaz gráfica revolucionaria con características como gráficos en color (cuatro colores: negro, blanco, azul, naranja), la multitarea preventiva, sonido estéreo y multi-estado de iconos (seleccionados y no seleccionados), siendo el antecedente de la Amiga 500, con su Workbench 1.3 que permitía además el uso de la televisión como monitor, 4096 colores, sistemas de color Pal y NTSC y sonido estero siendo un primer intento de integración entre las tecnologías digitales y la televisión, sentando las bases para la revolución del desktop video o video de escritorio en donde se podían fusionar a través de un dispositivo llamado Genlock, señales de una cámara de video o videograbadora con las generadas en la computadora en una pantalla de televisión, siendo de gran aplicación durante la década del 80 y principios de los 90 en productoras de contenido para televisión.

Ese año además, Bill Gates de la empresa Microsoft, que se dedicaba a desarrollar lenguajes 
como el BASIC que con instrucciones de palabras en ingles servían de lenguaje interprete entre el código máquina puro (sistema binario de ceros y unos) que manejan la computadora y el usuario, lanza Windows 1.0, su primer sistema operativo con iconos, aunque era muy inferior en el nivel de usabilidad para la gente a lo que ya existía en ese entonces en los prototipos de Macintosh y Amiga. Recién en 1992, con su versión de Windows 3.11 y sobre todo en 1995, con Windows 95 comienza a alcanzar más popularidad entre los usuarios.

\section{El inicio de la era tridimensional en las interfases y la integración digital}

En el año 1986, la empresa Silicon Graphics desarrolla un sistema operativo llamado Irix, que tenía 64 bits y una interfaz gráfica con iconos vectorizados (es decir gráficos basados en modelos matemáticos), que sentarían luego las bases de las gráficas tridimensionales facilitando a los usuarios el acceso a un espacio al otro lado de la pantalla que comienza a dejar el modelo bidimensional y gradualmente trata de replicar imágenes tridimensionales que son más familiares para el usuario común. Esto sumado al avance de la velocidad de los procesadores que permitían manejar un mayor contenido de información simultanea abre las puertas para la animación tridimensional y los desarrollo de interfases gráficas animadas.

En 1988, Steve Jobs tuvo la idea de crear una computadora que sirviera como prototipo de investigación entre universidades y laboratorios. Esta idea más tarde se convirtió en un proyecto llamado NeXT Computer y en 1989 desarrollo la interfase gráfica NeXT Step (Próximo paso) y luego el OpenStep cuyos iconos eran más grandes y con más cantidad de colores, siendo un interfaz moderna gracias al nivel gráfico que tenía, creando además en 1991, el Be Operation System (Ser Sistema Operativo), siendo el antecedente de la revolución posterior en el uso de aprovechar para el usuario la potencia de sumar muchas máquinas conectadas en red (capacidad multihilo), lo que sumado a la explosión de internet inició la expansión de la integración digital entre los usuarios.

\section{Desde el desarrollo de la interfase gráfica en los centros de investigación a la masividad}

Como en las visiones Orwellianas de la novela 1984, en donde el Hermano Mayor controla a través de la televisión la información que recibe la sociedad, el desarrollo de las interfases gráficas para los usuarios también precipitó una carrera en donde se pasó de las investigaciones entre laboratorios y universidades a la búsqueda del control de lo que los usuarios pueden realizar por medio de estas interfases desde sus computadores personales al acceder a internet. En esta dirección en 1995, Microsoft desarrolló el sistema operativo Windows 95 con una interfaz totalmente rediseñada con un sistema de navegación interno a través de "ventanas", y un navegador (Explorer) externo que vinculaba esta interfase con otras máquinas a través de la naciente Web comercial (sus inicios se dan en los 60 con fines militares a traves de la red Arpanet). Esta interfase gráfica que usaba la metáfora visual para el usuario de moverse por ventanas "internas" de la propia pc y "externas" de la web para ser navegadas, contaba con un botón "X" para cerrar estas ventanas, avanzando además en el concepto de tener diferentes "estados" (activado, desactivado) para los iconos brindándole un mayor control al usuario para producir 
diferentes acciones al otro lado de la pantalla dentro de los mundos virtuales.

Luego en 1997, Apple introduce los iconos isométricos (pseudo 3d) en el Mac OS System 8, y en 1998 con Windows 98 y el navegador de la web integrado Microsoft comienza a controlar el acceso desde las computadoras a Internet, dándole un poder extraordinario y monopólico a la compañía que para ese entonces superaba el $80 \%$ de computadoras (PC clones y de marca) que llevaban su sistema operativo instalado, a nivel mundial.

\section{La idea del acceso a la libertad de la información y el entorno Minix}

Usado en un sentido positivo este marco primigenio de navegación que es la interfase gráfica "interior", puede servir para facilitar la vida del usuario al realizar su trabajo, actividad lúdica, o comunicarse con otro mediante el acceso a la web por medio de la interfase gráfica "exterior. Por esta razón, grupos que ven como un riesgo a la libertad que una sola compañía posea un mercado tan grande, comenzaron a desarrollar interfases gráficas abiertas, en donde el código binario de base es libre de ser modificados por los usuarios para adaptarlos a sus necesidades específicas. Fue así que en 1987, el holandés Andrew Tanenbaum que estudio en Berkeley como Steve Jobs, desarrolló el Minix réplica gratuita de Unix con fines educativos para ser usado dentro de las redes universitarias, lo que posteriormente inspiró Linux un sistema operativo libre, creado por el ingeniero finlandes Linus Torvalds que se puede modificar, dando a la comunidad global en 1991 por medio de subir el código fuente al servidor de la universidades conectado a la web la versión 0.01 del sistema operativo Linux con 10.000 líneas de código, que llega en la actualidad a partir de las modificaciones realizadas por los usuarios a superar los 11 millones de líneas de código fuente.

Entre los principales desarrollos independientes basados en la interfase gráfica de Linux, tenemos al KDE 1.0, creado en 1998, que funcionaba bajo la metáfora de un escritorio amigable para estaciones de trabajo Unix, similar a los escritorios de MacOSX o Windows, pero con la gran ventaja de ser personalizable y de tener acceso al código fuente para ver lo que ocurre con la información en segundo plano y hacia donde va la misma, algo que era imposible de hacer en ese entonces con sistemas como el creado por Microsoft.

En la misma línea se desarrollo llego luego el proyecto GNOME que quiere decir GNU Network Object Model Environment (cuyo sentido aproximado sería Interfase gráfica orientada a modelos y objetos en ambientes de redes), originalmente desarrollado para Red Hat Linux, aunque después fue desarrollada para otras distribuciones de Linux y que buscaba hacer lo que ya hacían otros sistemas como Mac o Windows, pero dando el poder a los usuario sobre el control del desarrollo, algo que en una primera instancia si bien era posible, requería de personas que tuviesen un alto nivel de conocimientos y de alfabetización informática.

\section{La experiencia de navegación inmersiva y la interfase hacia el mundo virtual}

Ya comenzando el siglo XXI, los avances en el desarrollo de interfases gráficas han llevado a Steve Jobs desde 2001 en adelante a crear la interfaz Aqua que se va a alejando del modelo clásico de escritorio, para ingresar en la metáfora del mundo virtual, con zonas en donde las gráficas 
son transparentes (metafóricamente haciendo alusión a la idea de la transparencia de la información) y experiencias de navegación inmersivas tridimensionales con iconos animados que cobran "vida" al tocarlos, que evolucionarían luego en el sistema operativo Leopard en 2007 y luego en Tiger en la actualidad y en modelos abiertos en 3d como Beryl bajo Linux, o el intento de evolución de Windows XP, en Windows Vista, sin duda el mayor fracaso de Microsoft en la creación de entornos virtuales de trabajo. Sin embargo, el mundo digital siempre ofrece una oportunidad para la creatividad y estamos en los comienzos de una nueva revolución de la relación entre espectador e interfaz.

\section{Hacia las interfases alquímicas y los modelos lúdicos}

Sí la alquimia como concepto hace varios siglos, estuvo referido a experimentar si era posible transmutar una sustancia en otra, nosotros en el comienzo de este siglo XXI, estamos en los albores de transmutar los medios de comunicación conocidos a través de la integración digital, construyendo en cierto sentido "interfases alquímicas" de carácter híbrido que producirán la fusión de los diferentes campos de información gráficos, radiales, televisivos y virtuales a los que el espectador, usuario, observador, se vinculará de un modo posiblemente lúdico, aprendiendo a partir de ingresar y salir de los que hoy son medios diferentes, pero que en un futuro muy cercano estarán integrados.

En esta dirección, en el año 2008, Steve Ballmer y Bill Gates de la empresa Microsoft en la conferencia "Todo Digital" mostraron la nueva interfaz multitáctil que utiliza el nuevo sistema operativo Windows 7 y algo similar ocurrió con Snow Leopard por parte de Apple y dado la posición de dominación de ambos en el mercado global, sumado a los desarrollos futuros del papel digital (que reducirá la tala indiscriminada de bosques), del uso de interfase activadas por nuestros sentidos, usando nuestra visión, nuestra voz, nuestros oídos y nuestros pensamientos, potenciarán por un lado las interacciones sociales, pero, también, como contracara las operaciones y la construcción de simulacros que afecten estos ordenes sociales dentro del mundo virtual, por ejemplo poniendo en cuestionamiento el grado de veracidad de las noticias que recibimos cada día e incidiendo a partir de la dirección que éstas tomen el patrón de comportamiento de estos grupos.

\section{El simulacro virtual y el aporte a la construcción de la hiperrealidad}

Como mencionamos antes, los avances tecnológicos en los lenguajes virtuales conllevan el peligro de devenir en la construcción de hiperrealidades artificiales que el individuo termina creyendo. Según Jean Baudrillard, para construir nuestra identidad dependemos de la mirada del otro que nos objetiva, para lo cual en realidad todos nos transformamos en actores de un espectáculo. Esto se da porque la magia del mundo se desvanece ante el exceso de información sistemática, el éxtasis de las imágenes de las noticias cuya valoración a veces es cuestionable, a veces puede producir la "hipertrofia de la comunicación" -como diría Baudrillard- "acabando con toda mirada, con toda comunicación y reconocimiento...” (Vásquez Rocca, 2006).

Así, todo lo real es reducido a la mera apariencia. El sujeto trasciende a partir de la mirada del 
otro, se es para los otros. La manera en que somos vistos nos constituye, somos, a partir de un otro que nos seduce-nos mira- o al que seducimos-miramos.

Desde los medios a veces se exaltan muchas veces carentes de profundidad, creando personalidades prefabricadas para el consumo masivo, esto suele pasar frecuentemente en la moda y en la noción belleza artificial que esta construye cuando vemos en revista, televisión y otros medios modelos retocados mediante el lifting digital que, en general, la gente que no está al tanto de las nuevas tecnologías no puede reconocer, implantándole a muchas personas un ideal de belleza que en el fondo no existe.

En palabras de Baudrillard: "No hay un progreso continuo en esos ámbitos: la moda es arbitraria, pasajera, cíclica y no añade nada a las cualidades intrínsecas del individuo" (Vásquez Rocca, 2006).

Si esto funciona de este modo, se tiende a crear una hiperrealidad, una fantasía virtual de belleza eterna, una falsificación de la realidad, siendo posible que en el futuro el perfeccionamiento de los sistemas expertos y la tecnología de seguimiento de la futura "Web semántica" (web 3.0) contribuya a potenciar aún más este circuito, sino reflexionamos profundamente sobre esto, y si solo aceptamos estas tecnologías porque simplemente son "nuevas", sin comprender profundamente sus estructuras y sus implicancias, como ha pasado con la historia del desarrollo de las interfases gráficas.

\section{Nuevas tecnologías y vasos comunicantes}

La web 2.0, imaginada por Tim O'Reilly fue solo el comienzo, partiendo de la evolución de las aplicaciones tradicionales situadas en las máquinas de los usuarios hacia otras aplicaciones situadas en la web y enfocadas al usuario final. En este sentido el termino es más una actitud, una filosofía de ir hacia un lugar de integración, a partir de la noción de convergencia que reemplace el modelo anterior de interfase de escritorio, más que de una nueva tecnología para realizarlo. En la actualidad la Web 3.0, ha perfeccionado la tecnologías de seguimiento usando inteligencia artificial con programas inteligentes, que utilizan datos semánticos usando el la tecnología para la búsqueda a través de bases de datos en la red. La "Data Web" permite un nuevo nivel de integración de datos y aplicación interoperable, haciendo los datos tan accesibles y enlazables como las páginas web. La "Data Web" es el primer paso hacia la completa "Web Semántica”, de este modo, si creamos aplicaciones que produzcan razonamientos lógicos utilizando reglas que expresan relaciones entre conceptos y datos en la red y estos permiten por ejemplo estudiar que libros se lean más entre un grupo de personas de una comunidad, decodificar el perfil del libro, su estructuras, situaciones, etc. para que, luego, los sistema expertos en base al rastreo de Data Web, diseñen un libro a medida que se venda entre dicha comunidad.

Si bien esto es posible, desde el punto de vista teórico, aún falta tiempo. Antes que esto veremos que la convergencia de las distintas tecnologías mediante los vasos comunicantes que generen nuevos desarrollos en la web basados en el periodismo digital en blogs, los canales de usuarios en youtube, las nuevas formas publicitarias interactivas que detectan el perfil del navegante mandándole información a medida, el adsense, las nuevas culturas de twitters, flogers, flickers, etc sumadas a los avances en la robótica, los dispositivos táctiles del tipo Ipad, Iphone, las nuevas interfases gráficas mencionadas y la mayor disponibilidad de construcción de redes a nivel 
mundial con mayor ancho de banda, sumadas a la convergencia de televisión de la alta definición tridimensional, el video 360, la fusión con la telefonía celular multitactil y la internet inalámbrica en nuevos sistemas híbridos, darán al usuario mucho antes que el desarrollo real de la web 3.0, experiencias nuevas en los modos de circulación y comunicación de la información. Un aspecto clave en este sentido se dará a partir de la transmisión de la información en tiempo real de otras realidades simultaneas, pudiendo servir para crear por ejemplo oficinas virtuales, que le permitan interactuar simultáneamente con personas situadas en otros países para resolver problemas específicos, construir simulaciones virtuales en el campo de la psicología e ingresar dentro de ellas para ver como afectan la conducta humana ante determinadas situaciones, en medicina realizar operaciones simultaneas a muchos países a través de terminales de realidad virtual y en el campo del diseño nanométrico avanzar en el desarrollo de nuevos materiales y prototipos manipulando la materia a escala atómica.

\section{Conclusiones}

\section{Los nuevos modos de comunicación y los aportes para la construcción de una nueva trama social}

Asistimos a un Big Bang en los nuevos medios de comunicación a escala global, pero esto esta referido aún hoy a los grupos de investigación que producen esos medios, como estos se ofrecen y se consumen en el mercado.

Aún no existe, o no se vislumbra al menos, acuerdos a micro y macro escala comunitaria para aplicar con conciencia real y profunda estos nuevos medios en función de las necesidades que existen en cada sociedad.

Sin embargo tenemos una oportunidad. Sí lo enunciado en los párrafos previos es usado de un modo creativo y podemos recuperar el sentido lúdico que esta en el origen de toda creación humana podemos dar lugar a una explosión creativa a nivel social nunca antes vista, construyendo una nueva trama social que podrá incluir la aparición de nuevas profesiones, por ejemplo los Expertos en Medios Sociales (Social Media Expert - SME), modelos de conducta y mayores grados de libertad para todos.

Hoy los SME se dedican a hacer marketing en internet usando las redes sociales, sitios de actualización en tiempo real como Twitter y Blogs para ofrecer productos y servicios.

En general, estos expertos buscan generar el interés de los usuarios e intentan darle herramientas para que ellos mismos, por medio de sus opiniones, promocionen, divulguen entre sus referidos y generen discusiones sobre un producto, servicio o empresa en particular, buscando el factor naturalidad (en el sentido que los usuarios por iniciativa propia crean esas discusiones), que en realidad son inducidas por estos expertos en social media que en realidad están analizando como responde el consumidor frente a un determinado estímulo como si tratara de un experimento en un laboratorio social virtual, algo sobre lo que debemos reflexionar y cuestionar acerca de los alcances psicológicos de estas prácticas.

Como vemos en este análisis si bien disponemos de nuevos medios, aún las personas en general no han pasado por una alfabetización ordenada como para usarlos en un sentido positivo, más allá de las pruebas o experimentos sociales que puedan llevarse a cabo, ni existe una conciencia 
sobre los alcances y aplicaciones de estos medios ni siquiera en el área laboral.

Entre las acciones de tipo constructivo posibles, para lograr esta concientización por ejemplo, a nivel laboral, podría estar el racionalizar el tiempo disponible, dando a la gente la posibilidad de que el espacio se ocupe de diferente forma en nuestras ciudades, que las distancias se acorten y que las personas reduzcan su participación en actividades que requieran la presencia física, por dar un simple ejemplo, personas que viajan varias horas por día, para ir a sentarse frente a una terminal de computador, contaminando el medio ambiente, cuando pueden realizar la misma tarea desde una terminal móvil en cualquier parte del mundo.

Esto tendría, en principio, dos efectos sociales inmediatos y todo depende de como se mire, es decir si somos capaces de ver el vaso medio lleno o medio vacío. A priori, se podría pensar que las personas podrían estar más aisladas al conectarse de modo virtual en sus trabajos perdiendo relaciones con los otros, sin embargo estas mismas personas al tener más tiempo libre podrían destinarlo a contactar a otros grupos sociales, crear acciones comunitarias, participar de actividades en donde lo lúdico pueda dar paso a lo creativo potenciando las redes sociales virtuales y haciendo que estas dejen de ser virtuales para convertirse en acciones concretas en el mundo real. Si esto ocurre, posiblemente reforzaría los modos de comunicación entre los seres humanos, que hoy en día a pesar de los avances de la World Wide Web parecen estar en etapas de extinción, dado que la vorágine de la vida actual les resta tiempo a las personas para participar como seres sociales. De este modo, asistiremos al crecimiento de la inteligencia en red, dentro de una nueva trama o telaraña social, quizás a la llamada Web 4.0, en donde el concepto de inteligencia no solo éste aplicado a la resolución de problemas de orden lógico sino en encontrar otros caminos para divulgar la cultura y explorar nuevas formas de sensibilidad social entre los seres humanos.

\section{Notas}

1. Zhuang zi es uno de los fundadores del taoismo, movimiento filosófico iniciado por Lao-Tse durante el siglo V. Según esta visión, el tao es el fondo último del universo; el fondo invariable que late más allá de la pluralidad de las formas naturales.

\section{Lista de Referencias Bibliográficas}

Vásquez Rocca, A. (2006). Baudrillard; alteridad, seducción y simulacro, en PSIKEBA Revista de Psicoanálisis y Estudios Culturales. Buenos Aires.

Zhuang Zi (1996). Zhuang Zi. "Maestro Chuang Tsé” Traducción del chino a cargo de Iñaki Preciado Idoeta. Barcelona: Editorial Kairós.

\section{Bibliografía}

Carrol, L. (2003). Alicia en el país de las maravillas. A través del espejo. Traducción de Francisco Torres Oliver. Madrid: Akal Ediciones.

Gates, B. (1995). Camino al futuro. Madrid: Editorial McGraw-Hill. 
Gibson, W. (1996). Idoru. Editorial Minotauro.

Lao Tse Tao Te Ching. ( $2^{\circ}$ edición 2010). Los libros del Tao. Traducción directa del chino a cargo de Iñaki Preciado Idoeta. Madrid: Editorial Trotta.

Lessig, L. (1999). Las leyes del ciberespacio. Cuadernos Ciberespacio y Sociedad, No 3, Madrid. Negroponte, N. (1995). Ser digital. Barcelona: Ediciones Bailen.

Orwell, G. (2009). 1984. Colección: Áncora y Delfín. Barcelona: Ediciones Destino.

Rolando, F. L. (2005). La resignificación del espacio en la era de la virtualidad" en XIII Jornadas de Reflexión Académica en Diseño y Comunicación 2005. Formación de Profesionales Reflexivos en Diseño y Comunicación. Buenos Aires: Facultad de Diseño y Comunicación, Universidad de Palermo, pp. 198-206.

Vázquez Rocca, A, (2006). Baudrillard; alteridad, seducción y simulacro en PSIKEBA Revista de Psicoanálisis y Estudios Culturales. Buenos Aires.

Zhuang Zi (1996). Zhuang Zi. "Maestro Chuang Tsé" Traducción del chino a cargo de Iñaki Preciado Idoeta. Barcelona: Editorial Kairós.

\section{Recursos Electrónicos}

Lessig, L. Videoconferencia en Buenos Aires (2005). Disponible en: http://www.youtube.com/ watch? $=$ HkwIFuNjFXo

O’Reilly, T. Disponible en: http://radar.oreilly.com/

Vasquez Rocca, A. Disponible en: http://www.danoex.net/adolfovasquezrocca.html

Summary: The article focuses in the influence of virtuality in the real world, and considers the present stage on computing alphabetization, the interphase-user and the interface-spectator relations and how they have been changing in order to facilitate the social massive access to the new media, in a space known as cyberspace or hyperspace. The author intention is to analyze the interphase from its genesis, trying to explain the binary world and the ways to build the social hyperreality, and how it can affect the human being, who is influenced by the interaction with virtual reality that operates on the other side of the screens.

Key words: awareness - cyberspace - evolution - graphical interphase - hyperreality - mathematization - mutation - object - observer - relation - sensitivity - simulation - social framework - virtual networks - ways - web 2.0 - web 3.0.

Resumo: $\mathrm{O}$ trabalho se centra na influência do virtual no mundo real, considerando o estádio atual da alfabetizão informática, a relação interfase-usuário e interface - espectador e como foi mudando para facilitar o acceso em massa das sociedades aos novos modos de comunicação e circulação da informação ao outro lado das telas, numa zona conhecida como ciberespaço ou hiperespaço considerando como isto poderá devir na construção de novas tramas sociais, mas também afetar suas condutas.

Para isto se partirá em tratar de analisar o problema da interfase desde sua génese, tratando de explicar o mundo binário e as formas em que este pode construir o que denominamos Hi- 
perrealidade Social, como isto pode afetar-nos socialmente, quando o sujeito de estudo, neste caso o ser humano, vê-se influído pela interação que tem como usuário mediante o uso das interfase gráficas e a realidade virtual que opera ao outro lado das telas (por trás do cristal) numa zona que chamaremos ciberespaço, tratando de propor nas conclusões que aportes construtivos podem fazer-se ao problema proposto.

Palavras chave: caminhos - ciberespaço - concientização - evolução - hiperrealidade - interfase gráfica - matematización - mutação - objeto - observador - redes virtuais - relação - sensibilidade - simulacro - trama social - web 2.0 - web 3.0. 